\title{
Fast spectral fitting of hard X-ray bremsstrahlung from truncated power-law electron spectra
}

\author{
J. C. Brown ${ }^{1}$, J. Kašparová ${ }^{2}$, A. M. Massone ${ }^{3}$, and M. Piana ${ }^{4,3}$
}

\author{
1 Department of Physics and Astronomy, University of Glasgow, Glasgow G12 8QQ, UK \\ e-mail: john@astro.gla.ac.uk \\ 2 Astronomický ústav AV ČR, v.v.i., Fričova 298, 25165 Ondřejov, Czech Republic \\ 3 CNR-INFM LAMIA, via Dodecaneso 33, 16146 Genova, Italy \\ e-mail: michele.piana@univr.it \\ ${ }^{4}$ Dipartimento di Informatica, Università di Verona, Ca' Vignal 2, Strada le Grazie 15, 37134 Verona, Italy \\ Received 1 February 2008 / Accepted 31 May 2008
}

ABSTRACT

\begin{abstract}
Context. Hard X-ray bremsstrahlung continuum spectra, such as from solar flares, are commonly described in terms of power-law fits, either to the photon spectra themselves or to the electron spectra responsible for them. In applications various approximate relations between electron and photon spectral indices are often used for energies both above and below electron low-energy cutoffs.

Aims. We examine the form of the exact relationships in various situations, and for various cross-sections, showing that empirical relations sometimes used can be highly misleading especially at energies below the low-energy cutoff, and consider how to improve fitting procedures.

Methods. We obtain expressions for photon spectra from single, double and truncated power-law electron spectra for a variety of cross-sections and for the thin and thick target models and simple analytic expressions for the non-relativistic Bethe-Heitler case. Results. We show that below the low-energy cutoff Kramers and other constant spectral index forms commonly used are very poor approximations to accurate results, but that our analytical forms are a good match; and that above a low-energy cutoff, the Kramers and non-relativistic Bethe-Heitler results match reasonably well with results for up to energies around $100 \mathrm{keV}$.

Conclusions. Analytical forms of the non-relativistic Bethe-Heitler photon spectra from general power-law electron spectra are good match to exact results for both thin and thick targets and they enable much faster spectral fitting than evaluation of the full spectral integrations.
\end{abstract}

Key words. Sun: flares - Sun: X-rays, gamma rays - methods: data analysis

\section{Introduction}

Hard X-ray bremsstrahlung spectra are important diagnostics of flare electron acceleration and propagation - e.g., Brown (1971); Lin \& Schwartz (1987); Johns \& Lin (1992); Thompson et al. (1992); Piana (1994); Holman et al. (2003). Extensive use of this diagnostic power has been enabled by the high resolution spectra being observed by RHESSI (Lin et al. 2002), which handles very large spectral and dynamic ranges. At low energies (a few keV for microevents and up to 20 or so $\mathrm{keV}$ for large flares), the spectrum is usually consistent with isothermal bremsstrahlung - e.g. Holman et al. (2003) - while at higher energies it is usually consistent with bremsstrahlung from a (sometimes broken) powerlaw electron spectrum with a low-energy cutoff. Broadly similar forms, such as the shifted power law $\left(E+E_{\mathrm{s}}\right)^{-\delta}$ (with $E_{\mathrm{s}}$ a shifting energy value), can also be consistent with the data in many cases. One should bear in mind that the data $I(\epsilon)$ to be considered are those after application of corrections for the albedo spectrum contribution (Kontar et al. 2006) as well as instrumental effects. Here we focus on the properties of the deka-keV energy power-law domain, especially truncated power-law forms (including broken power-laws) since these are so widely used and discuss the relationship between the "local" spectral indices $\delta(E)$ of the source electrons and $\gamma(\epsilon)$ of the observed photons. In data analysis it is quite common (e.g. Hannah et al. 2008) to assume constant values of $\gamma(\epsilon)$ and of $\delta(E)$ over specific finite energy ranges and definite linear relationships between $\gamma$ and $\delta$ in these
- for example, in the energy ranges below and above the electron cutoff. However, as we show below, for general bremsstrahlung cross-sections, most such relationships are at best approximate and the various ad hoc relationships used can be quite misleading. Our aim is to show the exact form of these relationships for various cross-sections and derive analytic expressions simple enough for easy use in fast spectral analysis software.

For both the thin- and thick-target models this is accomplished by first showing numerically that the non-relativistic Bethe-Heitler approximation for the bremsstrahlung crosssection is reliable enough to reproduce quite well the true photon spectrum corresponding to a truncated power-law electron spectrum. Then for both models we obtain exact analytical expressions for $I(\epsilon)$ and $\gamma(\epsilon)$ based on the non-relativistic Bethe-Heitler cross-section. Finally these expressions are used to best fit simulated and measured photon spectra and determine electron spectrum parameters. The effectiveness of this method is assessed by comparisons with two different fitting approaches currently employed: (1) fitting with a numerical expression for $I(\epsilon)$ obtained by full numerical integration (Holman et al. 2003) of truncated power-law electron spectra $\bar{F}(E)$ and $\mathcal{F}_{0}\left(E_{0}\right)$ (thin and thick target, respectively); (2) fitting data on $I(\epsilon)$ with a broken powerlaw characterized by two distinct constant photon spectral indices, one above and one below a "knee" energy (e.g. Hannah et al. 2008). With respect to the first approach, we find that our new method yields comparably good $\chi^{2}$ values and residuals especially at lower energies but with substantially higher 
computational speed (much higher in the case of thick targets). With respect to the second approach, our method is no faster but much more accurate and meaningful. In fact the $I(\epsilon)$ used in approach (2) is unphysical, corresponding to no real $\bar{F}(E)$ so it may give excessive $\chi^{2}$ values due to large residuals near the knee.

Section 2 provides the general equations for bremsstrahlung spectra for both collisionally thin and thick target sources. Section 3 establishes our notation for a power-law $\bar{F}(E)\left[\mathcal{F}_{0}\left(E_{0}\right)\right]$ of constant $\delta\left(\delta_{0}\right)$ truncated below the low-energy cutoff $E_{1}$ $\left(E_{01}\right)$, and shows how arbitrarily truncated and multiple (broken) power-laws can be expressed in terms of these. Section 4 defines the Kramers and non-relativistic Bethe-Heitler approximate bremsstrahlung cross-sections and contrasts the results they give for $I(\epsilon)$ and $\gamma(\epsilon)$ for power-law electron spectra compared with that for the fully relativistic cross-section. In Sects. 5 and 6 we obtain analytic expressions, in both thin- and thicktarget cases, for $I(\epsilon)$ and $\gamma(\epsilon)$. In Sect. 7 we report numerical tests of the speed and accuracy of using these to fit real and simulated data, as compared with other approximate methods and with full integration. Section 8 summarises our conclusions.

\section{Thin and thick target bremsstrahlung and energy losses}

For a general inhomogeneous optically thin source of plasma density $n(\boldsymbol{r})$ and electron flux energy spectrum $F(E, \boldsymbol{r})$ in volume $V$, the bremsstrahlung photon flux energy spectrum $I(\epsilon)\left(\mathrm{cm}^{-2} \mathrm{~s}^{-1}\right.$ per unit $\epsilon$ at Earth distance $\left.R\right)$ can be written (Brown 1971):

$I(\epsilon)=\frac{\bar{n} V}{4 \pi R^{2}} \int_{\epsilon}^{\infty} \bar{F}(E) Q(\epsilon, E) \mathrm{d} E$,

with

$\bar{n}=\int_{V} n \mathrm{~d} V / V$

and

$\bar{F}(E)=\int_{V} n(\boldsymbol{r}) F(E, \boldsymbol{r}) \mathrm{d} V /(\bar{n} V)$

where $Q(\epsilon, E)$ is the bremsstrahlung cross-section differential in photon energy $\epsilon$. In general, $\bar{F}(E)$ and $Q(\epsilon, E)$ have to be treated as anisotropic and Eq. (1) involves an integral over solid angle (Brown 1972; Massone et al. 2004) though most data treatments assume source isotropy.

In a purely collisional thick target, $\bar{F}(E)$ is related to the thick-target injection rate spectrum $\mathcal{F}_{0}\left(E_{0}\right)$ (electrons per second per unit injection energy $\left.E_{0}\right)$ through equation

$\bar{F}(E)=\frac{1}{K \bar{n} V} E \int_{E}^{\infty} \mathcal{F}_{0}\left(E_{0}\right) \mathrm{d} E_{0}$

regardless of $Q(\epsilon, E)$, with $K=2 \pi \mathrm{e}^{4} \Lambda$ and $\Lambda$ the Coulomb logarithm - Brown \& Emslie (1988). Though they consider only the case $K=2 \pi \mathrm{e}^{4} \Lambda$ for collisional losses only in a uniformly ionized target, Eq. (4) applies to any energy loss rate coefficient $K(E)$ if the loss rate can be written in the form $\mathrm{d} E / \mathrm{d} N=$ $-K(E) / E$ where $N$ is the column density along the electron path such as for collisional losses at high $E$ with relativistic correction. Synchrotron losses cannot be written in this way unless the magnetic field and electron pitch angle distribution do not vary along the path, but these also only matter at high energies. The most serious approximation involved in using constant $K$ for lower energies is in (common) neglect of the fact that $K$ varies with target hydrogen ionization $x$ (Brown 1973; Emslie 1978) being $K=2 \pi \mathrm{e}^{4} \Lambda(1+a x) /(1+a)$ with $a \approx 1.6$. This is important around the energies of electrons $E_{*} \approx\left(2 K N_{*}\right)^{1 / 2}$ stopping around the flare transition zone column density $N_{*}$, where atmosphere ionization drops rather abruptly with consequent drop in energy loss coefficient $K$ (for details of the effect of this on the hard X-ray spectra, see Brown 1973; Kontar et al. 2003). Apart from early stages of flares, prior to much evaporation, $E_{*}$ is well above typical values of the low-energy cutoff considered here. Since we are concerned mainly with small $E$ around low-energy cutoff values, henceforth we address only the case of constant $K$ and take $\Lambda=25$.

\section{Single and broken power-laws}

We consider first the widely used single power-law form with low-energy cutoff for the thin target $\bar{F}(E)$

$\bar{F}(E)=(\delta-1) \frac{F_{1}}{E_{1}}\left\{\begin{array}{cl}{\left[\frac{E}{E_{1}}\right]^{-\delta}} & E \geq E_{1} \\ 0 & E<E_{1}\end{array}\right.$

where $F_{1}=\int_{E_{1}}^{\infty} \bar{F}(E) \mathrm{d} E$ is the total mean electron flux at $E \geq$ $E_{1}$, a low-energy cutoff ${ }^{1}$, and $\delta$ is the (thin target; Brown 1971) constant electron spectral index.

Before considering the photon spectral properties of such single power-law electron spectra we note that our results for these can easily be generalised to fitting of double (broken) power-laws in $\bar{F}(E)$ with lower and upper cutoff energies. The following decomposition expressions apply equally well to any broken and truncated power-laws such as in photon space $I(\epsilon)$. The general case is

$\bar{F}(E)=\left\{\begin{array}{lr}0 & E<E_{\mathrm{c}} \\ A E^{-\delta_{1}} & E_{\mathrm{c}} \leq E<E_{\mathrm{b}} \\ A E_{\mathrm{b}}^{-\delta_{1}+\delta_{2}} E^{-\delta_{2}} & E_{\mathrm{b}} \leq E<E_{\mathrm{a}} \\ 0 & E \geq E_{\mathrm{a}}\end{array}\right.$

where $A, E_{\mathrm{c}}, E_{\mathrm{b}}, E_{\mathrm{a}}, \delta_{1}, \delta_{2}$ are constants (we note that in the general case (6) the low-energy cutoff is denoted with $E_{\mathrm{c}}$ ). Writing the parameterized single power-law $F_{\mathrm{pl}}\left(c, d, E^{*}\right)$ as

$F_{\mathrm{pl}}\left(c, d, E^{*}\right)=\left\{\begin{array}{lr}c E^{-d} & E \geq E^{*} \\ 0 & \text { otherwise }\end{array}\right.$

$\bar{F}(E)$ in (6) can be always written as

$$
\begin{aligned}
\bar{F}(E)= & F_{\mathrm{pl}}\left(A, \delta_{1}, E_{\mathrm{c}}\right)-F_{\mathrm{pl}}\left(A, \delta_{1}, E_{\mathrm{b}}\right) \\
& +F_{\mathrm{pl}}\left(A E_{\mathrm{b}}^{-\delta_{1}+\delta_{2}}, \delta_{2}, E_{\mathrm{b}}\right)-F_{\mathrm{pl}}\left(A E_{\mathrm{b}}^{-\delta_{1}+\delta_{2}}, \delta_{2}, E_{\mathrm{a}}\right) .
\end{aligned}
$$

By inserting Eq. (8) into Eq. (1), the corresponding $I(\epsilon)$ can be found simply as the sum and difference of the relevant $I(\epsilon)$ expressions for single power-laws.

For the thick target model, we have to revisit the problem and evaluate the form of $\bar{F}(E)$ and hence of $I(\epsilon)$ for a truncated power law in $\mathcal{F}_{0}\left(E_{0}\right)$, not in $\bar{F}(E)$. Note also that we have to distinguish between the spectral index $\delta_{0}$ for a pure power-law injection spectrum $\mathcal{F}_{0}\left(E_{0}\right)$ from the index $\delta$ for $\bar{F}(E)$. For an injection spectrum truncated at $E_{0} \leq E_{01}$

$\mathcal{F}_{0}\left(E_{0}\right)= \begin{cases}\left(\delta_{0}-1\right) \frac{\mathcal{F}_{01}}{E_{01}}\left[\frac{E_{0}}{E_{01}}\right]^{-\delta_{0}} & E_{0} \geq E_{01} \\ 0 & E_{0}<E_{01},\end{cases}$

\footnotetext{
1 If one wishes to use an electron flux reference energy $E_{*}$ distinct from the cutoff energy $E_{1}$ one must replace $F_{1}$ in Eq. (5) by $F_{*}\left(E_{*} / E_{1}\right)^{-\delta+1}$.
} 
where $\mathcal{F}_{01}=\int \mathcal{F}\left(E_{0}\right) \mathrm{d} E_{0}$ and Eq. (4) gives

$\bar{F}(E)=\frac{\mathcal{F}_{01} E_{01}}{K \bar{n} V}\left\{\begin{array}{cl}{\left[\frac{E}{E_{01}}\right]^{-\delta_{0}+2}} & E \geq E_{01} \\ \frac{E}{E_{01}} & E<E_{01},\end{array}\right.$

i.e., the relation between $\delta_{0}$ and $\delta$ at $E \geq E_{01}$ being $\delta=\delta_{0}-2$. To find $\bar{F}(E)$ from a broken and truncated power-law form of the injected thick target $\mathcal{F}_{0}\left(E_{0}\right)$, one would first use the analogy of expression (8) to get the total $\mathcal{F}_{0}\left(E_{0}\right)$ as the sum of a set of single power-law $\mathcal{F}_{0}\left(E_{0}\right)$ forms, insert it into Eq. (4) and use expression (10) for each term in the sum to get the corresponding $\bar{F}(E)$. Then, such $\bar{F}(E)$ is inserted into Eq. (1) to obtain the corresponding $I(\epsilon)$ for the broken power-law thick-target case.

\section{Cross-sections}

The bremsstrahlung cross-section $Q(\epsilon, E)$ can be written as

$Q(\epsilon, E)=\frac{Q_{0} m c^{2}}{\epsilon E} q(\epsilon, E)$,

incorporating a high $Z$ element correction factor $\sum_{Z} A_{Z} Z^{2}$ in $Q_{0}$, the Gaunt factor $q(\epsilon, E)$ depending on the actual cross-section used. For the Kramers approximation

$q(\epsilon, E)=q_{K}(\epsilon, E)=1$;

while for the non-relativistic Bethe-Heitler approximation

$q(\epsilon, E)=q_{\mathrm{BH}}(\epsilon, E)=\log \frac{1+\sqrt{1-\epsilon / E}}{1-\sqrt{1-\epsilon / E}}$.

The most widely used isotropic formula (neglecting electronelectron bremsstrahlung which is important at high energies Kontar et al. 2007) is $q(\epsilon, E)=q_{3 \mathrm{BN}}(\epsilon, E)$, corresponding to the fully relativistic Bethe-Heitler 3BN formula from Koch \& Motz (1959). This formula (or its corrected version including Elwert factor) is used in inversion and forward fits in Piana et al. (2003); Kontar et al. (2005); Brown et al. (2006); Johns \& Lin (1992). From hereafter we will refer to the non-relativistic Bethe-Heitler Eq. (13) as the Bethe-Heitler formula and to the fully relativistic Bethe-Heitler formula 3BN in Koch \& Motz (1959) with Elwert factor included as the $3 \mathrm{BN}$ formula. In the case of a truncated power-law, the sensitivity of the predicted thin- and thick-target photon spectra to the form of the cross-section used is illustrated in Fig. 1 by numerically computing the local photon spectral index, defined by Brown \& Emslie (1988) as

$\gamma(\epsilon)=-\frac{\epsilon}{I} \frac{\mathrm{d} I}{\mathrm{~d} \epsilon}=-\frac{\mathrm{d} \log I}{\mathrm{~d} \log \epsilon}$,

for single power-laws with $\delta=3, \delta=5 ; \delta_{0}=5, \delta_{0}=7$ and various $q$. In Fig. 1 we also show the percentage difference in photon fluxes defined as

$\Delta I(\epsilon)=\frac{I_{3 \mathrm{BN}}(\epsilon)-I_{\mathrm{BH}}(\epsilon)}{I_{3 \mathrm{BN}}(\epsilon)}$,

where $I_{3 \mathrm{BN}}$ is the photon flux using $q_{3 \mathrm{BN}}$ and $I_{\mathrm{BH}}$ corresponds to the photon flux using $q_{\mathrm{BH}}$. It is clear firstly that, particularly below the cutoff, Bethe-Heitler results are a rather good approximation for computing local photon spectral indices $\gamma(\epsilon)$ and photon fluxes. In particular, the relative error in the computation of the photon flux is between $10 \%$ and $15 \%$ around the low-energy cutoff, much smaller at lower energies and grows up to $20 \%$ only for energies larger than $100 \mathrm{keV}$. Second, above and especially below the cutoff $\gamma(\epsilon)$ is not in any way constant, as sometimes assumed (Hannah et al. 2008).

As a further check, Fig. 2 demonstrates the behaviour of $\gamma(\epsilon)$ and $\Delta I(\epsilon)$ for a broken power-law in the electron space. Figures 1 and 2 thus clearly suggest that the analytical expressions of $I(\epsilon)$ and $\gamma(\epsilon)$ based on the Bethe-Heitler formula could be useful for spectral computation in forward spectral fitting, especially at low energies. At energies approaching $100 \mathrm{keV}$ the approximation is poorer and it needs to be used with some caution in locating features like high energy cutoffs, though these are always uncertain even for exact computation - cf. Brown et al. (2006).

Note that Fig. 1 shows results for only one value of $E_{1}\left(E_{01}\right)=20 \mathrm{keV}$ and presents them only as functions of $\epsilon / E_{1}$ $\left(\epsilon / E_{01}\right)$ whereas one might in general expect results to depend on $\epsilon$ and $E_{1}\left(E_{01}\right)$ separately (an analogous situation occurs in Fig. 2, where the results are shown only for one value of $E_{\mathrm{c}}$ ). However, we carried out a range of test calculations for several different $E_{1}\left(E_{01}\right)$ in the few deka-keV range and found that results were, to a very good approximation, functions only of $\epsilon / E_{1}$ $\left(\epsilon / E_{01}\right)$ at these energies. Second, the most general $Q$ is actually anisotropic and the above expressions have to be generalised to integrate over electron angle as well as energy (Massone et al. $2004)$. However, the effect of this on $I(\epsilon)$ is small at low energies and in any case is mainly an $\epsilon$-independent scaling rather than a spectral effect. In fact some numerical experiments showed that there is little effect on our conclusions of using anisotropic $\bar{F}, Q$.

\section{Thin target spectra and spectral indices}

Using Eq. (11), Eq. (1) becomes

$I(\epsilon)=\frac{Q_{0} m c^{2}}{4 \pi R^{2}} \frac{\bar{n} V}{\epsilon} \int_{\epsilon}^{\infty} \bar{F}(E) q(\epsilon, E) \frac{\mathrm{d} E}{E}$.

We are interested here in results for photon spectra $I(\epsilon)$ when $\bar{F}(E)$ is a single power-law with constant $\delta$ and a lowenergy cutoff (Eq. (5)), for various forms of $q(\epsilon, E)$, for comparison of $\gamma(\epsilon)$ with the use (e.g. Hannah et al. 2008) of constant $\gamma$ approximations. For any $q(\epsilon, E)$ Eq. (16) can be written

$I(\epsilon)=\frac{\delta-1}{\delta} C \frac{E_{1}}{\epsilon} \int_{0}^{\min \left[1,\left(E_{1} / \epsilon\right)^{\delta}\right]} q\left(\epsilon, E_{1} / x^{1 / \delta}\right) \mathrm{d} x$

where $x=\left(E_{1} / E\right)^{\delta}$, and

$C=\frac{Q_{0} m c^{2}}{4 \pi R^{2}} \frac{\bar{n} V F_{1}}{E_{1}^{2}}$

Note that in special cases where $q(\epsilon, E)=q(\epsilon / E)$ only, $\gamma(\epsilon)$ takes the form

$\gamma(\epsilon)= \begin{cases}\delta+1 & \epsilon \geq E_{1} \\ 1-\frac{\mathrm{d}}{\mathrm{d} \log \epsilon} \log \left[\int_{0}^{1} q\left(x^{1 / \delta} \epsilon / E_{1}\right)\right] \mathrm{d} x & \epsilon<E_{1} .\end{cases}$

\subsection{Kramers cross-section}

For Kramers $q(\epsilon, E)=1$ and we have immediately

$I(\epsilon)=\frac{\delta-1}{\delta} C \frac{E_{1}}{\epsilon}\left\{\begin{array}{cl}{\left[\frac{E_{1}}{\epsilon}\right]^{\delta}} & \epsilon \geq E_{1} \\ 1 & \epsilon<E_{1}\end{array}\right.$

and

$\gamma(\epsilon)=\left\{\begin{array}{cl}\delta+1 & \epsilon \geq E_{1} \\ 1 & \epsilon<E_{1}\end{array}\right.$ 


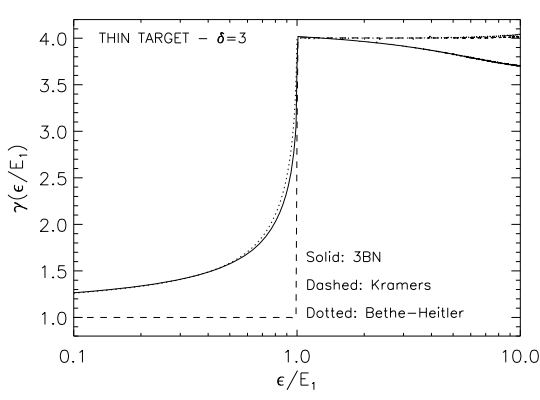

(a)

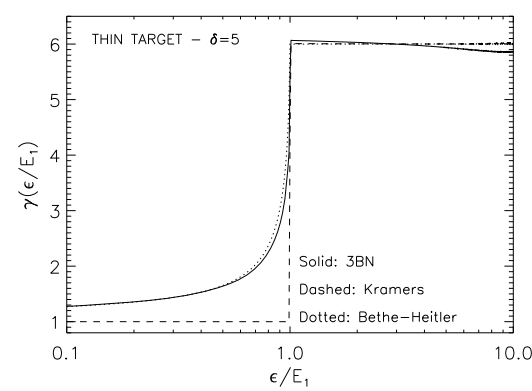

(c)

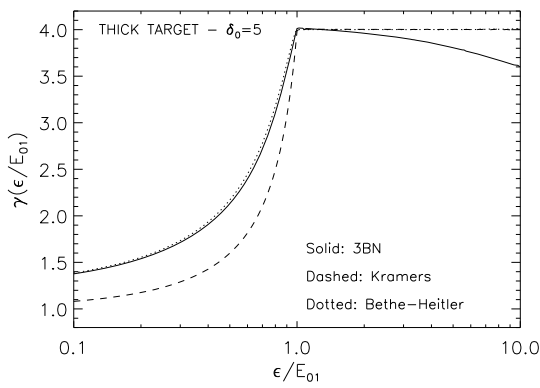

(e)

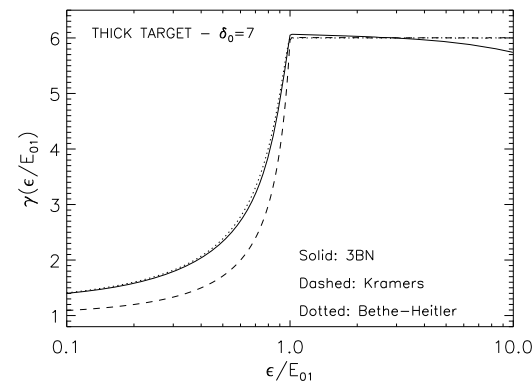

(g)

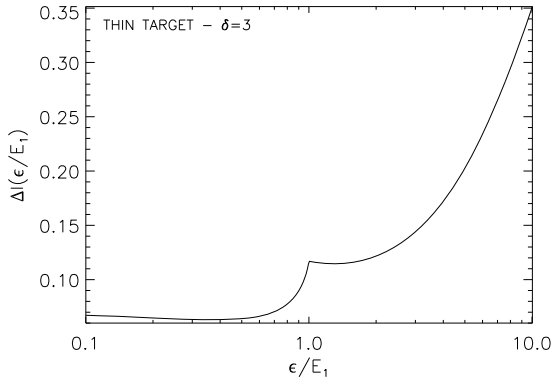

(b)

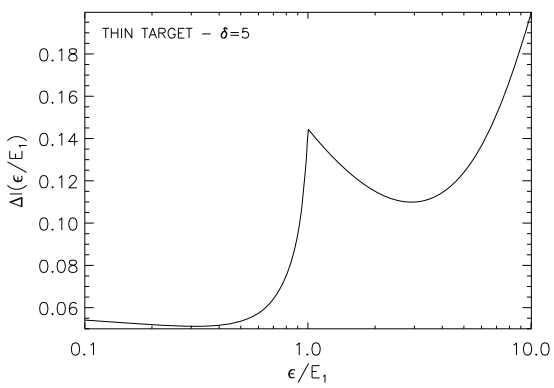

(d)

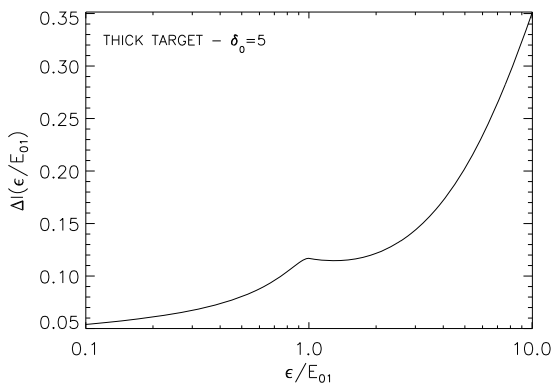

(f)

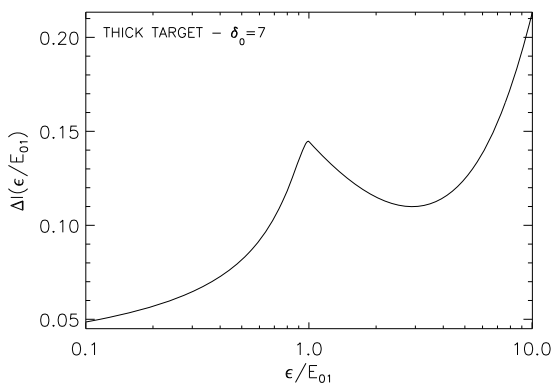

(h)
Fig. 1. Left panels: local bremsstrahlung photon spectral index $\gamma(\epsilon)$ behaviour computed numerically for three different $q(\epsilon, E)$ - Kramers, Bethe-Heitler and $3 \mathrm{BN}$ and a single power-law with $E_{1}\left(E_{01}\right)=20 \mathrm{keV}$. Right panels: corresponding percentage difference $\Delta I(\epsilon)$ in photon flux as defined in Eq. (15). a) and b) Thin target with $\delta=3$. c) and d) Thin target with $\delta=5$. e) and f) Thick target with $\delta_{0}=5$. g) and h) Thick target with $\delta_{0}=7$.

\subsection{Bethe-Heitler cross-section}

The spectrum and the spectral index can be written solely in terms of the electron spectral index $\delta$ and the dimensionless parameter $a=\epsilon / E_{1}$. Integration by parts leads to:

$I(\delta, a)=\frac{\delta-1}{\delta} C \begin{cases}\frac{1}{a^{\delta+1}} B\left(\delta, \frac{1}{2}\right) & a \geq 1 \\ \frac{1}{a}\left[\log \frac{1+\sqrt{1-a}}{1-\sqrt{1-a}}+\frac{1}{a^{\delta}} B_{a}\left(\delta, \frac{1}{2}\right)\right] & a<1\end{cases}$

with $B(\alpha, \beta)=\int_{0}^{1} x^{\alpha-1}(1-x)^{\beta-1} \mathrm{~d} x$ and $B_{x}(\alpha, \beta)=\int_{0}^{x} t^{\alpha-1}(1-$ $t)^{\beta-1} \mathrm{~d} t$ the incomplete beta function.

For the spectral index:

$\gamma(\delta, a)= \begin{cases}1+\delta & a \geq 1 \\ 1+\frac{\frac{\delta}{a^{\delta}} B_{a}\left(\delta, \frac{1}{2}\right)}{\log \left[\frac{1+\sqrt{1-a}}{1-\sqrt{1-a}}\right]+\frac{1}{a^{\delta}} B_{a}\left(\delta, \frac{1}{2}\right)} & a<1 .\end{cases}$

We note that the previous analytical formulas for $I(\delta, a)$ and $\gamma(\delta, a)$ are written in terms of beta functions and incomplete beta functions. The computation of these functions is included in standard library routines for data visualization and analysis, making the implementation of the exact formulas (22) and (23) easy and fast. 


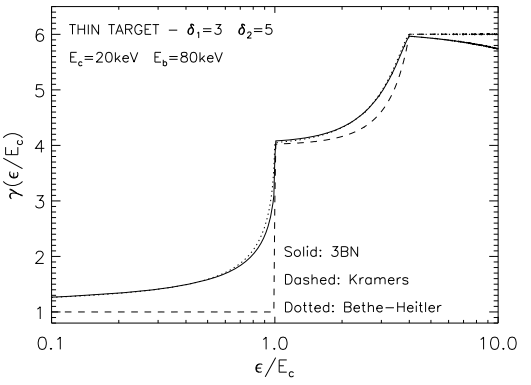

(a)

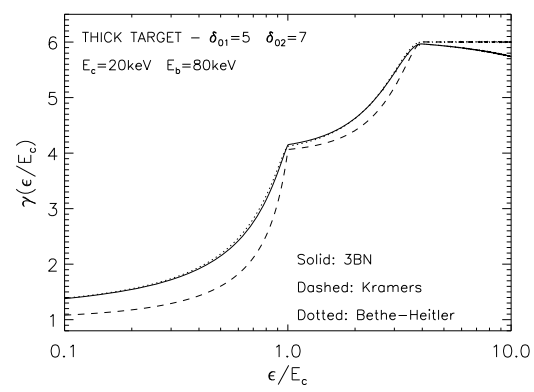

(c)

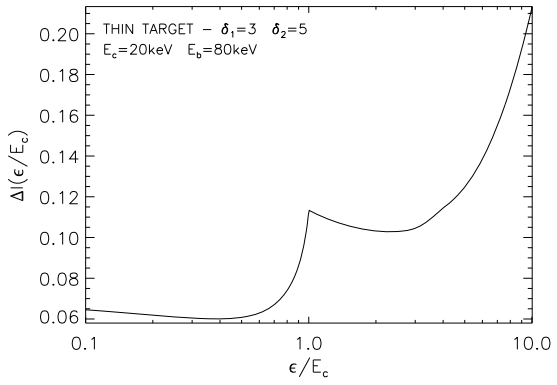

(b)

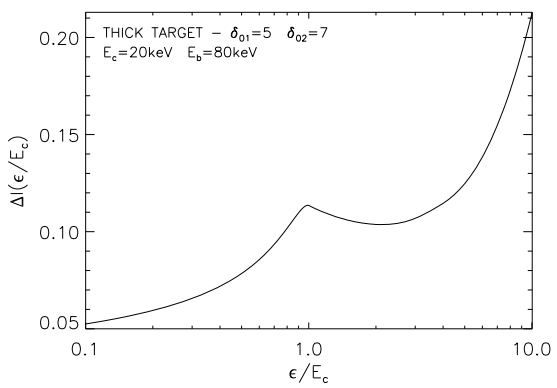

(d)
Fig. 2. The same as in Fig. 1 but for a broken power-law with $E_{\mathrm{c}}=20 \mathrm{keV}$ and $E_{\mathrm{b}}=80 \mathrm{keV}$. a) and b) Thin target with $\delta_{1}=3, \delta_{2}=5$. c) and d) Thick target with $\delta_{01}=5, \delta_{02}=7$.

\section{Thick target spectra and spectral indices}

Inserting (10) into (16) leads to

$I(\epsilon)=\frac{D}{\delta_{0}-2} \frac{E_{01}}{\epsilon} \times\left\{\begin{array}{l}\int_{0}^{\left(\frac{E_{01}}{\epsilon}\right)^{\delta_{0}-2}} q\left(\epsilon, E_{01} / y^{\frac{1}{\delta_{0}-2}}\right) \mathrm{d} y \quad \epsilon \geq E_{01} \\ \int_{0}^{1} q\left(\epsilon, E_{01} / y^{\frac{1}{\delta_{0}+2}}\right) \mathrm{d} y+ \\ \left(\delta_{0}-2\right) \int_{\epsilon / E_{01}}^{1} q\left(\epsilon, \xi E_{01}\right) \mathrm{d} \xi \quad \epsilon<E_{01},\end{array}\right.$

where $D=\frac{Q_{0} m c^{2} \mathcal{F}_{01}}{4 \pi R^{2} K}$, the integrals on the right hand side of (24) being expressed below in terms of the dimensionless parameter $a=\epsilon / E_{01}$, the constant $D$ and the spectral index $\delta_{0}$ for the truncated electron power-law $\mathcal{F}_{0}\left(E_{0}\right)$.

\subsection{Kramers cross-section}

Integration by parts with Kramers unity Gaunt factor gives

$I\left(\delta_{0}, a\right)=\frac{D}{\delta_{0}-2} \begin{cases}a^{-\delta_{0}+1} & a \geq 1 \\ \frac{1+\left(\delta_{0}-2\right)(1-a)}{a} & a<1\end{cases}$

and

$\gamma\left(\delta_{0}, a\right)= \begin{cases}\delta_{0}-1 & a \geq 1 \\ \frac{\delta_{0}-1}{1+\left(\delta_{0}-2\right)(1-a)} & a<1 .\end{cases}$

\subsection{Bethe-Heitler cross-section}

Integration by parts with the Bethe-Heitler Gaunt factor $q_{\mathrm{BH}}$ leads to

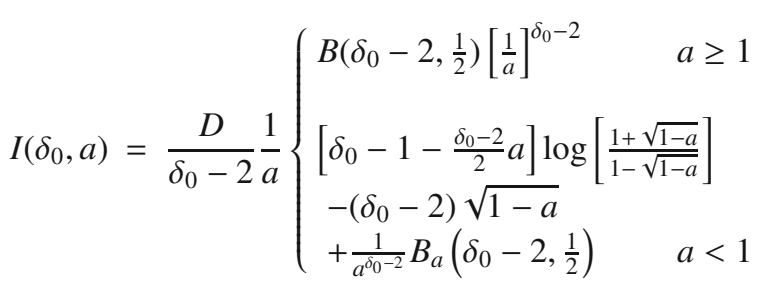

and

$$
\gamma\left(\delta_{0}, a\right)= \begin{cases}\delta_{0}-1 & a \geq 1 \\ \frac{A_{1}+A_{2} B_{a}\left(\delta_{0}-2, \frac{1}{2}\right)}{A_{3}-\sqrt{1-a}+A_{4} B_{a}\left(\delta_{0}-2, \frac{1}{2}\right)} & a<1,\end{cases}
$$

where

$$
\begin{aligned}
& A_{1}=A_{1}\left(\delta_{0}, a\right)=\frac{\delta_{0}-1}{\delta_{0}-2} \log \frac{1+\sqrt{1-a}}{1-\sqrt{1-a}} \\
& A_{2}=A_{2}\left(\delta_{0}, a\right)=\frac{\delta_{0}-1}{\delta_{0}-2} \frac{1}{a^{\delta_{0}-2}}, \\
& A_{3}=A_{3}\left(\delta_{0}, a\right)=\left(\frac{\delta_{0}-1}{\delta_{0}-2}-\frac{a}{2}\right) \log \frac{1+\sqrt{1-a}}{1-\sqrt{1-a}}
\end{aligned}
$$

and

$A_{4}=A_{4}\left(\delta_{0}, a\right)=\frac{1}{\delta_{0}-2} \frac{1}{a^{\delta_{0}-2}}$.

Thus, as in the thin target case, integral expressions for $I\left(\delta_{0}, a\right)$ and $\gamma\left(\delta_{0}, a\right)$ can be written analytically in terms of beta functions and incomplete beta functions.

\section{Tests against data and comparison with other fitting methods}

To show the usefulness of our formulation in terms of accuracy and speed, we have tried it out on simulated and real data. Our specific goals here for simulated noisy data are to determine:

1. how good and how fast is use of our functional approximations to $I(\epsilon)$ in fitting data to estimate $\left[\delta, \bar{n} V F_{1}, E_{1}\right]$ or $\left[\delta_{0}, \mathcal{F}_{01}, E_{01}\right]$ for a [thin] or [thick] target single truncated power-law $[\bar{F}(E)]$, or $\left[\mathcal{F}_{0}\left(E_{0}\right)\right]$, in comparison with other fitting routines. To answer this we use simulated data $I(\epsilon)$ generated with NASA SolarSoft (SSW) routines using the full bremsstrahlung cross-section and numerical integration over $E$ or $E_{0}$, respectively (Holman et al. 2003); 
Table 1. Computational cost test. The test was performed on a $3.2 \mathrm{GHz}$ PC with 1GB RAM. Times are in seconds.

\begin{tabular}{ccccc}
\hline \hline & SSW-thick & Eq. (27) & SSW-thin & Eq. (22) \\
\hline $\begin{array}{c}\delta, \delta_{0}=3 \\
E_{1}, E_{01}=10\end{array}$ & 7.45 & 0.721 & 1.27 & 0.726 \\
$\quad \delta, \delta_{0}=3$ & & & & \\
$\begin{array}{c}E_{1}, E_{01}=20 \\
\delta, \delta_{0}=3\end{array}$ & 7.32 & 0.594 & 0.951 & 0.726 \\
$\begin{array}{c}E_{1}, E_{01}=30 \\
\quad \delta, \delta_{0}=5\end{array}$ & 9.99 & 0.585 & 0.931 & 0.599 \\
$\begin{array}{c}E_{1}, E_{01}=10 \\
\quad \delta, \delta_{0}=5\end{array}$ & 9.99 & 0.721 & 1.36 & 0.724 \\
$\begin{array}{c}E_{1}, E_{01}=20 \\
\delta, \delta_{0}=5\end{array}$ & 7.46 & 0.594 & 1.07 & 0.595 \\
$E_{1}, E_{01}=30$ & 7.05 & 0.583 & 1.20 & 0.597 \\
\hline
\end{tabular}

2. how well do the speed and accuracy compare with other approaches? In particular with: (a) Holman et al. (2003) who, in carrying out the best fit parameter searches, perform full integrations with the full cross-section in each iterative step; and (b) an approach which, instead of fitting the $I(\epsilon)$ predicted for an electron power-law with cutoff, fits a parametric piecewise power-law photon $I(\epsilon)$ with distinct constant photon spectral indices $\gamma_{1}, \gamma_{2}$ at $\epsilon \leq, \geq \epsilon_{\mathrm{b}}$, with $\gamma_{1}$ either a free fit parameter or prescribed, e.g. as in Hannah et al. (2008) where $\gamma_{1}=1.5$ (to be compared with $\gamma(\epsilon)$ in Fig. 1). Loosely speaking, for example in the case of thin targets, the value of the photon break energy $\epsilon_{\mathrm{b}}$ is meant to reflect an electron low-energy cutoff energy $E_{1}$, though in reality such a photon spectrum does not correspond to any real (non-negative) electron spectrum except for Kramers cross-section in which case $\gamma_{1}=1, \gamma_{2}=\delta+1$;

3 . how each of the above findings changes when we add a reasonable isothermal contribution to $I(\epsilon)$ for thermal parameters $E M$ and $T$.

We have carried out these simulated data comparisons for the following parameter sets: $\delta, \delta_{0}=3,5, E_{1}, E_{01}=10,20,30 \mathrm{keV}$, $\bar{n} V F_{1}=5,20 \times 10^{55}$ electrons $\mathrm{cm}^{-2} \mathrm{~s}^{-1} ; \mathcal{F}_{01}=5,20 \times 10^{35}$ electrons s $\mathrm{s}^{-1}, E M=0.5,1 \times 10^{49} \mathrm{~cm}^{-3}$, and $k T=1,1.5 \mathrm{keV}$.

$I(\epsilon)$ were generated using SSW routines for thin and thick target ( $f_{-}$thin. pro and $f_{-}$thick . pro, respectively) and optionally with isothermal component (f_vth.pro) in the 3-100 keV energy range with $1 \mathrm{keV}$ energy binning. Then, these $I(\epsilon)$ were converted to counts using the RHESSI detector response matrix for attenuator state 0 . Finally, we added Poisson noise. Such simulated count spectra were then fitted within the OSPEX environment ${ }^{2}$ in the $3-100 \mathrm{keV}$ range.

The results of comparisons for the simulated spectra without a thermal component are as follows:

1. for both thin and thick cases, acceptable fits in terms of reduced $\chi^{2}, \chi_{v}^{2}$, and normalised residuals were found for all simulated spectra using expressions (22) and (27), respectively. The fitted parameters were close to the input ones within $\sim 10 \%$ or less.

Concerning the computational time (see Table 1) for the fitting procedures, the SSW thin fit routine was about 2 times slower than our method for similar accuracy while the SSW thick fit routine is 10-20 times slower than our method;

\footnotetext{
2 http://hesperia.gsfc.nasa.gov/ssw/packages/spex/doc/ ospex_explanation.htm
}

2. as regards the matter of trying to get a meaningful fit to the actual form of $I(\epsilon)$ from truncated power-laws $F(E), \mathcal{F}_{0}\left(E_{0}\right)$ by using double power-law fits to the photon spectra (constant $\gamma$ above and below some break energy $\epsilon_{\mathrm{b}}$ - bpow .pro) we found that this failed to produce an acceptable overall fit (generally, $\chi_{v}^{2}>2$ ) and that normalised residuals clustered near $\epsilon \sim E_{1}, E_{01}$. Using a fixed value of $\gamma_{1}$ such as $1,1.5,1.7$ did not help.

Adding a plausible isothermal component with the above values for parameters $E M$ and $T$ to the thin and thick target spectra modifies the fit behaviour described above only if the thermal component contributes significantly to or dominates the spectrum at $\epsilon \gtrsim E_{1}, E_{01}$. For such spectra, e.g. thin-target case $E M=1 \times 10^{49} \mathrm{~cm}^{-3}, k T=1.5 \mathrm{keV}, \delta=3, E_{1}=10 \mathrm{keV}$, the fitting functions introduced in this paper do give an acceptable fit but only an upper limit on $E_{1}$ can be obtained. This limit is close to the energy where the thermal spectrum steepens and falls below the non-thermal part, fits with smaller $E_{1}$ being also consistent with the data since lost in the dominant thermal emission.

Photon spectra with a thermal component can also usually be fitted with a nonthermal component close to a single powerlaw so the value of $\epsilon_{\mathrm{b}}$ obtained by double photon power law fits is not a good indicator of $E_{1}, E_{01}$. Depending on the combination of thermal and non-thermal parts, acceptable fits using bpow yield $\epsilon_{\mathrm{b}}$ which can be either lower or higher than the input $E_{1}, E_{01}$. Therefore, using $\epsilon_{\mathrm{b}}$ for an estimate of the non-thermal energy (e.g. Hannah et al. 2008) can be misleading. On the other hand, our expressions generally give $E_{1}, E_{01}$ much closer to the input $E_{1}, E_{01}$ than $\epsilon_{\mathrm{b}}$ is.

The proposed expressions have been also tested on two cases of real data. Figure 3 shows thin and thick target fits to the 20-Feb.-2002 11 UT flare and compares the parameters obtained by our and SSW expressions. Both thin and thick fits give simi$\operatorname{lar} \bar{F}(E)$ and $\mathcal{F}_{0}\left(E_{0}\right)$. This flare was near the limb so required no albedo correction.

Next, we applied our thick target expression to the early impulsive phase of the flare of 02-Jun.-2002 which shows flattening and evidence of a low-energy cutoff at $E_{01}$ above the thermal component in the $18-38 \mathrm{keV}$ range (Sui et al. 2007). In this case albedo correction was applied, as is essential for such events. Figure 4 shows the time evolution $E_{01}(t)$ obtained for the time variation of the best fit low-energy cutoff and for the corresponding total non-thermal electron power (as total energy per $4 \mathrm{~s}$ integration). These curves are closely comparable with those found by Sui et al. (2007) in their Fig. 4.

Thin and thick target formulas (Eqs. (22), (27)) have been incorporated into the SSW tree. Prospective users may access them as OSPEX fitting functions named photon_thin.pro and photon_thick.pro.

\section{Conclusions}

We have shown that results for thin and thick target bremsstrahlung photon spectra $I(\epsilon)$ and $\gamma(\epsilon)$ from power-law electron spectra with constant index $\delta$ and low-energy spectral cutoff $E_{1}, E_{01}$ obtained using the non-relativistic Bethe-Heitler cross-section are close to those from the exact cross-section, at photon energies both above and below the cutoff, at least at $\mathrm{keV}$ to deka-keV energies. We have shown further that the nonrelativistic Bethe-Heitler expression allows the bremsstrahlung integrals for $I(\epsilon)$ to be written as analytic forms in terms of beta functions of $\delta\left(\delta_{0}\right)$ and $a$ only, which are part of standard 

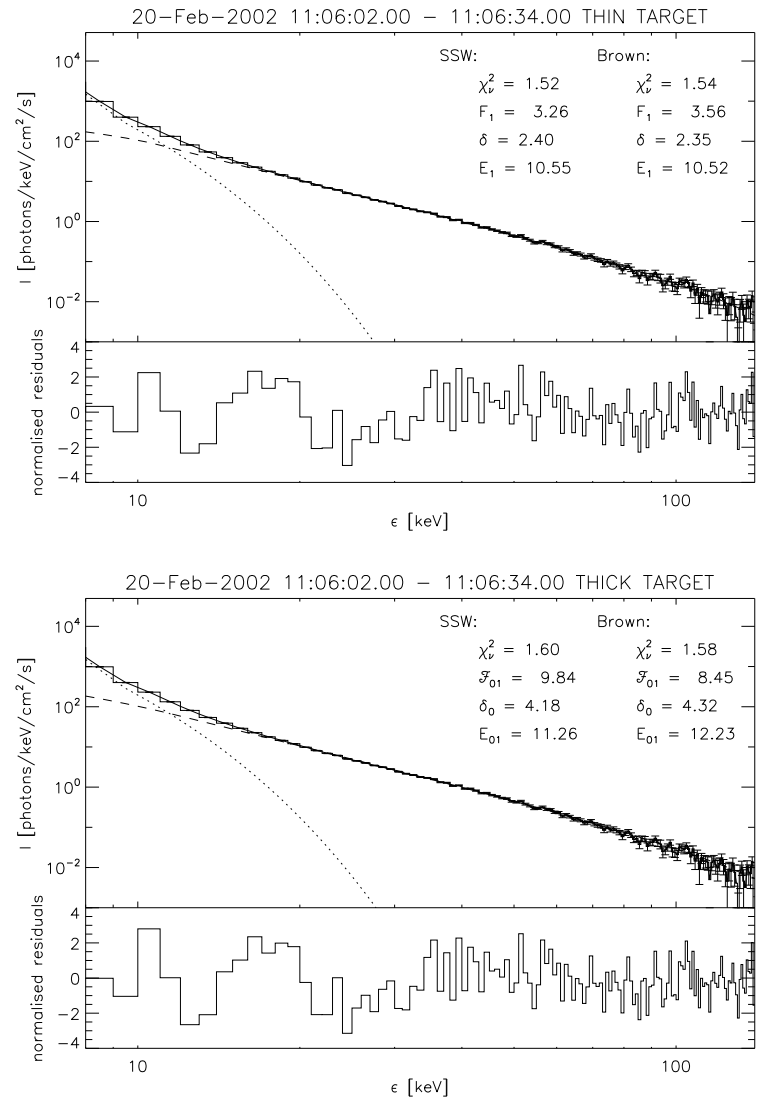

Fig. 3. Thin and thick target fits to February 20, 2002 flare spectrum using our expressions. The labels show the comparison between SSW and our approach.

numerical packages, and that these give results very close to the exact $I(\epsilon)$.

For both the thin and especially for the thick target models, we find that this formulation enables almost equally accurate but much faster spectral fitting than evaluation of the full spectral integrations. This will be valuable in analysis of bremsstrahlung HXR data such as from RHESSI, providing, for example, a fast method to obtain first approximation to $\bar{F}(E)$ or $\mathcal{F}_{0}\left(E_{0}\right)$ for input to inversion methods. Our analytic fast fit approach can also replace the unphysical practice of fitting broken power-laws in $I(\epsilon)$.

Acknowledgements. We kindly acknowledge financial support from ISSI, UK STFC and UC Berkeley SSL (J.C.B.), the Italian MIUR, grant I/015/07/0 of the Italian ASI/INAF (AMM and MP), grant 205/06/P135 of the Grant Agency
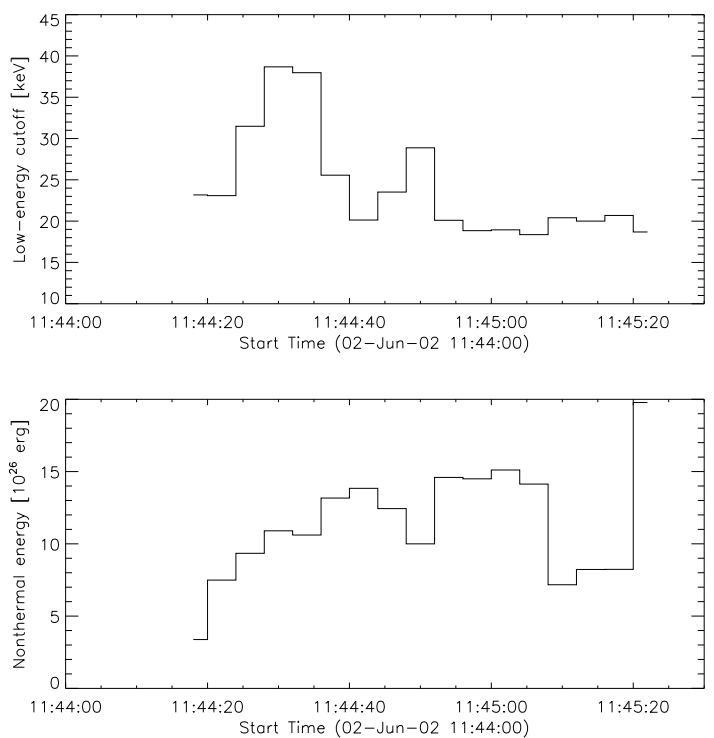

Fig. 4. Low-energy cutoff and total non-thermal electron energy in 4s time intervals for the June 22002 flare as derived from our approach, see also Fig. 4 in Sui et al. (2007).

of the Czech Republic and key project AV0Z10030501 (J.K.). The paper has benefited from discussions with H.S. Hudson, A. Caspi and P. Saint-Hilaire.

\section{References}

Brown, J. C. 1971, Sol. Phys., 18, 489

Brown, J. C. 1972, Sol. Phys., 26, 441

Brown, J. C. 1973, Sol. Phys., 29, 421

Brown, J. C., \& Emslie, A. G. 1988, ApJ, 331, 554

Brown, J. C., Emslie, A. G., Holman, G. D., et al. 2006, ApJ, 643, 523

Emslie, A. G. 1978, ApJ, 224, 241

Hannah, I. G., Christe, S., Krucker, S., et al. 2008, ApJ, 677, 704

Holman, G. D., Sui, L., Schwartz, R. A., \& Emslie, A. G. 2003, ApJ, 595, L97

Johns, C. M., \& Lin, R. P. 1992, Sol. Phys., 137, 121

Koch, H. W., \& Motz, J. W. 1959, Rev. Mod. Phys., 31, 920

Kontar, E. P., Brown, J. C., Emslie, A. G., et al. 2003, ApJ, 595, L123

Kontar, E. P., Emslie, A. G., Piana, M., Massone, A. M., \& Brown, J. C. 2005, Sol. Phys., 226, 317

Kontar, E. P., MacKinnon, A. L., Schwartz, R. A., \& Brown, J. C. 2006, A\&A, 446, 1157

Kontar, E. P., Emslie, A. G., Massone, A. M., et al. 2007, ApJ, 670, 857

Lin, R. P., \& Schwartz, R. A. 1987, ApJ, 312, 462

Lin, R. P., Dennis, B. R., Hurford, G. J., et al. 2002, Sol. Phys., 210, 3

Massone, A. M., Emslie, A. G., Kontar, E. P., et al. 2004, ApJ, 613, 1233

Piana, M. 1994, A\&A, 288, 949

Piana, M., Massone, A. M., Kontar, E. P., et al. 2003, ApJ, 595, L127

Sui, L., Holman, G. D., \& Dennis, B. R. 2007, ApJ, 670, 862

Thompson, A. M., Brown, J. C., Craig, I. J. D., \& Fulber, C. 1992, A\&A, 265, 278 